\title{
Editorial
}

\section{Reduced-Order Modeling, Testing, and Control of Nonlinear Mechanical and Structural Systems}

\author{
José Manoel Balthazar, ${ }^{1}$ Paulo Batista Gonçalves, ${ }^{2}$ Angelo Marcelo Tusset, ${ }^{3}$ \\ Grzegorz Litak, ${ }^{4}$ and Livija Cveticanin ${ }^{5}$ \\ ${ }^{1}$ Aeronautics Technological Institute, São José dos Campos, SP, Brazil \\ ${ }^{2}$ Pontifical Catholic University, Rio de Janeiro, RJ, Brazil \\ ${ }^{3}$ Federal University of Technology-Parana, Ponta Grossa, PR, Brazil \\ ${ }^{4}$ Lublin University of Technology, Lublin, Poland \\ ${ }^{5}$ University of Novi Sad, Novi Sad, Serbia
}

Correspondence should be addressed to José Manoel Balthazar; jmbaltha@ita.br

Received 9 July 2017; Accepted 16 July 2017; Published 16 August 2017

\begin{abstract}
Copyright ( 2017 José Manoel Balthazar et al. This is an open access article distributed under the Creative Commons Attribution License, which permits unrestricted use, distribution, and reproduction in any medium, provided the original work is properly cited.
\end{abstract}

This special issue addresses the general problem of model size reduction for describing nonlinear vibration of structural elements and systems. The aim is to provide and analyze tools that allow accurately capturing nonlinear dynamic behavior using a minimal number of degrees of freedom. It involves modeling and control of nonlinear dynamical phenomena in structural systems and other complex multibody mechanisms. The problems discussed involve identification and control of nonlinear systems as well as responses of the nonlinear system under cyclic excitations. The problem is related to the dimensionality of the nonlinear system which could be a measure of complexity. It is worth noticing that in nonlinear systems there are a variety of multiple solutions which, together with a synchronization phenomenon, play basic and many times competing roles. In some of the systems, for example, the systems showing stick and slip phenomenon, due to dry friction, the dimensionality varies in time. On the other hand, nonlinear systems with memory are characterized by long transients which strongly influence the dynamics identification control procedure. Such a memory effect is frequently observed as a hysteretic behavior based on appearing additional degrees of freedom and can be modeled with a fractional derivative. Finally, the material, structural, and multibody interaction nonlinearities can lead to localized effects which increase the system complexity but the particular response of the dynamical system can be described by a size reduced model.

The authors of one of the papers study the chaotic oscillations in a fractional order model of a portal frame with nonideal loading. The bifurcation of the fractional order portal frame system for parameters and fractional orders are investigated. Adaptive sliding mode controllers are designed to suppress the chaotic oscillations with uncertain parameters. In another paper, the authors predict the pullout capacity of small ground anchors using nonlinear computing techniques. Results show that the developed prediction models enhance the precision of predicting the pullout capacity when compared with previous studies. In one of the papers, in order to investigate the seismic damage of cable-stayed bridges, a scaled model of a single-tower cable-stayed bridge with A-shaped tower was designed, constructed, and tested on shake tables. Experimental and simplified numerical analysis show the correspondence with fairly small error. One of the papers is dealing with dynamic nonlinear responses and stability of slender structures in the main resonance regions. In this paper, an incremental technique for direct calculation of the nonlinear response in frequency domain of plane frames subjected to base excitation was proposed. In another paper a control methodology is applied to an automated manual transmission vehicle for drivability enhancement 
during vehicle start-up phase. Based on a piecewise model of powertrain, a multiple-model predictive controller (mMPC) is designed with the engine speed, clutch disc speed, and wheel speed as the measurable input variables and the engine torque reference and clutch friction torque reference as the controller's output variables. In another paper fatigue tests were performed on plain concrete under triaxial tensioncompression-compression (T-C-C) cyclic loading with constant and variable amplitude using a large multiaxial machine. Experimental results show that, under constant amplitude fatigue loads, the development of residual strain in the fatigue loading direction depends mostly on the lateral compressive stress ratio and is nearly independent of stress level. In one of the papers, a new reduced-order controller based on the improved Balanced Truncation (BT) method is designed to reduce the calculation time and to retain the abandoned high-order modal information. In addition, a design method based on Guaranteed Cost Control (GCC) algorithms was presented to eliminate the adverse effects of data acquisition and actuator response time-delays in the design process of the reduced-order controller. A paper is devoted to a novel electric Gibbs function proposed for the piezoelectric microbeams (PMBs) by employing a modified couple stress theory. Based on the new Gibbs function and the Euler-Bernoulli beam theory, the governing equations that incorporate the effects of couple stress, flexoelectricity, and piezoelectricity were derived for the mechanics of PMBs. In another paper, the bearing fault signal was detected adaptively, and time-frequency characteristics of the fault signal can be extracted accurately. The validity of this method was verified by the identifications of simulated shock signal and test bearing fault signal. The method provides a new understanding of wavelet spectrum detection based on spectral kurtosis for rolling element bearing fault signal. Finally, in one of the papers, in order to identify the quadratic Volterra system simplified from the hydroturbine shaft system, a blind identification method based on the third-order cumulants and a reversely recursive method were proposed. The input sequence of the system under consideration is an unobservable independent identically distributed, zero-mean, and non-Gaussian stationary signal, and the observed signals are the superposition of the system output signal and Gaussian noise. The results show that the method is applicable to the blind identification of the hydroturbine shaft system and has strong universality.

José Manoel Balthazar

Paulo Batista Gonçalves Angelo Marcelo Tusset Grzegorz Litak Livija Cveticanin 


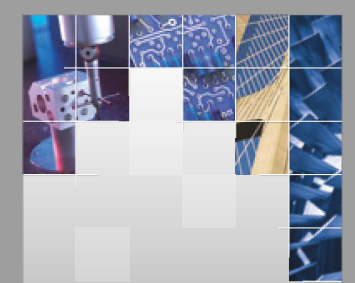

\section{Enfincering}
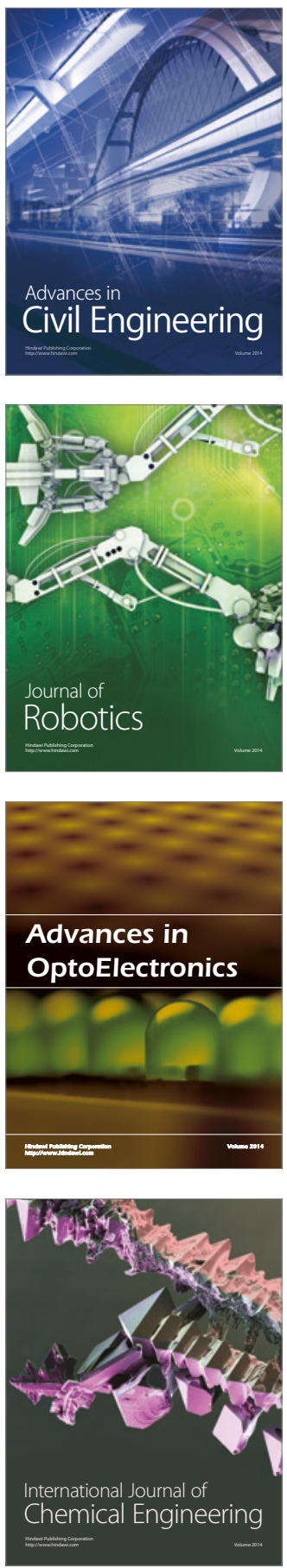

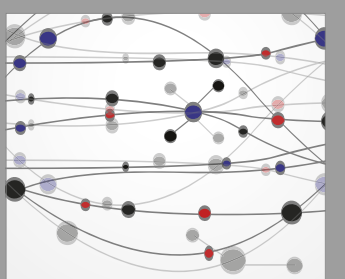

The Scientific World Journal

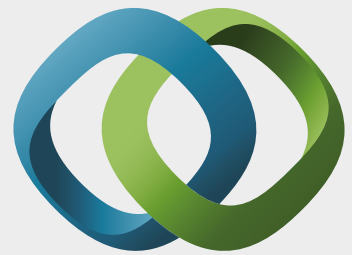

\section{Hindawi}

Submit your manuscripts at

https://www.hindawi.com
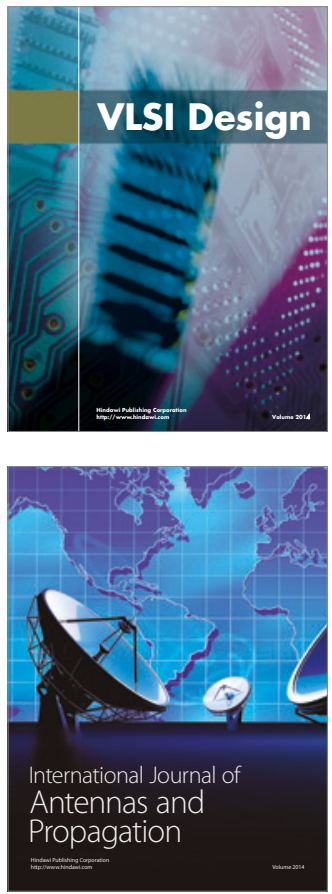

\section{Rotating}

Machinery
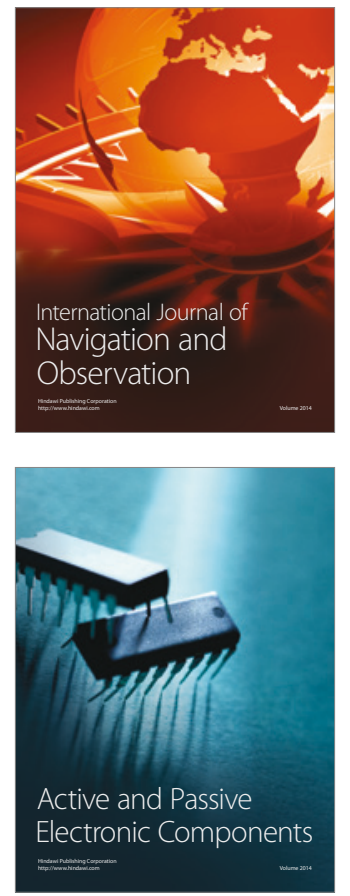
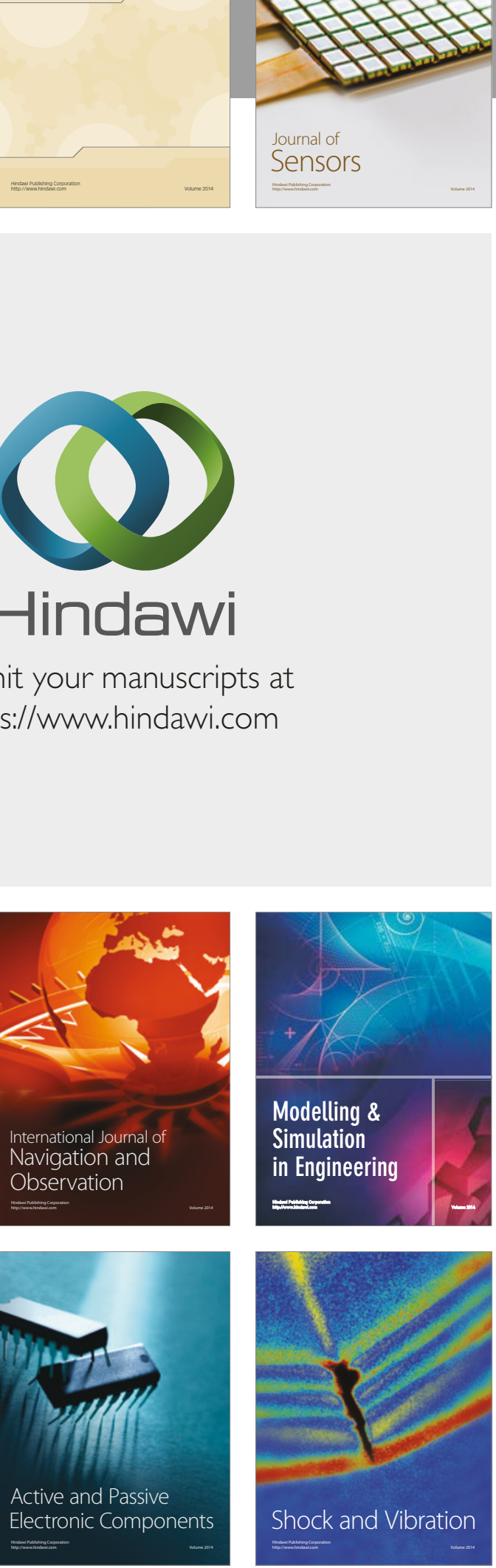
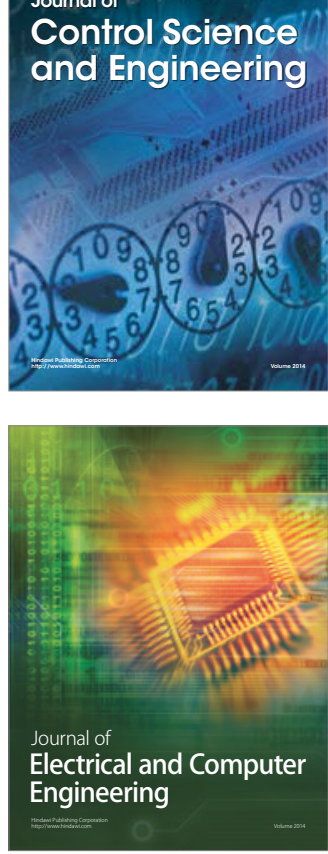

Distributed

Journal of

Control Science

and Engineering
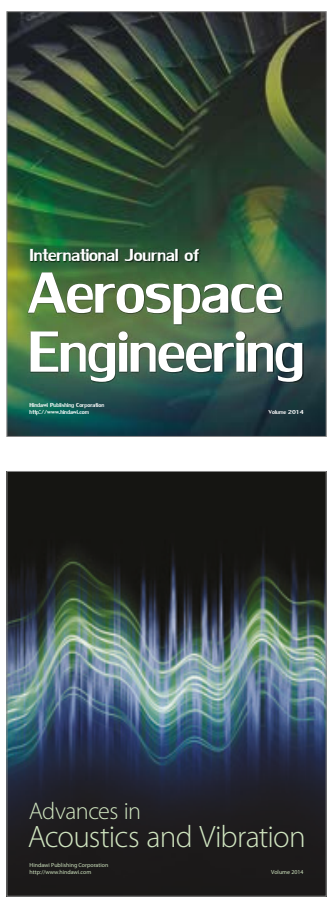

Sensor Networks 